\title{
Data Processing and Analysis Method of NLOS Ranging Radar System
}

\author{
Huixian Jiang \\ Key Laboratory of Specialty Fiber Optics and Optical \\ Access Networks, Shanghai University \\ Shanghai, China \\ circle0324@126.com
}

\author{
Guoxin Zheng, Zhongpin Luo, Xiao Chen \\ Key Laboratory of Specialty Fiber Optics and Optical \\ Access Networks, Shanghai University \\ Shanghai, China \\ gxzheng@staff.shu.edu.cn
}

\begin{abstract}
We address the problem of precise ranging between trains in non-line-of-sight (NLOS) environment using a NLOS (Non Line of Sight) ranging radar. By calculating the phase delay of the received and transmitted OFDM (Orthogonal Frequency Division Multiplexing) waveform, we achieve the target of ranging in NLOS environment. Then, we compare several different data processing methods and prefer equal mean value weighted coefficient method. In addition, we analyze the ranging error according to the characteristics of the AWGN channel, thereby providing a theoretical basis for the development of the OFDM waveform phase ranging system.
\end{abstract}

Keywords- NLOS, ranging, data processing, equal mean value weighted coefficient

\section{INTRODUCTION}

"Shanghai 9.27" subway crash shows that when the communication signal system fails, an active ranging radar device is needed between trains. The existing ranging radar in accordance with different working patterns can be divided into two kinds: active and responsive. As is known that, millimeter wave and ultrasonic radars attenuate easily thus can only be used in short distance ranging, while pulse radar works only in the line-of-sight environment. However, there are only two kinds of radars based on the responsive working principle, the deep space exploration and marine rescue, which can realize the distance ranging in NLOS environment, but only in long distance with low accuracy. Therefore, we propose a NLOS [1-2] ranging radar in this article, which applies the principle of a continuous phase ranging and the responsive radar, thereby increasing the ranging accuracy in short-distance NLOS environment.

The existing radio-ranging technologies are pseudorandom code ranging, phase ranging and OFDM-based ranging [3]. Although Pseudo-random code ranging has the advantages of anti-interference, anti-noise and anti-multipath attenuation, its ranging accuracy is very low. Phase ranging has a higher ranging accuracy. However, its ranging range is restricted due to the period of cycle. OFDM-based ranging combines the superiority of the above two methods effectively. In time domain, a method similar to pseudorandom code ranging is used to acquire maximum unambiguous distance range. In frequency domain, ranging is achieved through the phase difference of any two subcarries. Based on this, this article analyzes the statistical regularities of various harmonics of OFDM waveforms. We hypothesize that the mean value is unequal while the variance is equal. The conventional equal weighed method can't minimize the measurement error. Moreover, the existing OFDM-based ranging increases the variance by subtracting different harmonics values, which will lower the measuring accuracy. In this article, we analyze and compare different data processing methods and finally apply equalaverage weighted coefficient method to NLOS ranging radar system, thereby achieving the most accurate ranging result. The structure of this article is as follows: (1) Introduction (2) NLOS radar system (3) Data processing method (4) The four-wire carrier measurement errors (5) Variance and the signal-to-noise ratio relationship (6) Conclusions.

\section{NLOS RADAR SYSTEM}

\section{A. Operation Principle}

As is shown in Fig. 1, the frequency-shift processing time is $\Delta t^{\prime \prime}$, the phase delay time is $\Delta t^{\prime}$.Firstly, train A generates a RF signal and transmits it to train $\mathrm{B}$. Then, train $\mathrm{B}$ retransmits it. Finally, train A demodulates it and calculates the actual transmitting time $\Delta t$. $\left(\Delta t=\Delta t^{\prime}-\Delta t^{\prime \prime}\right)$.

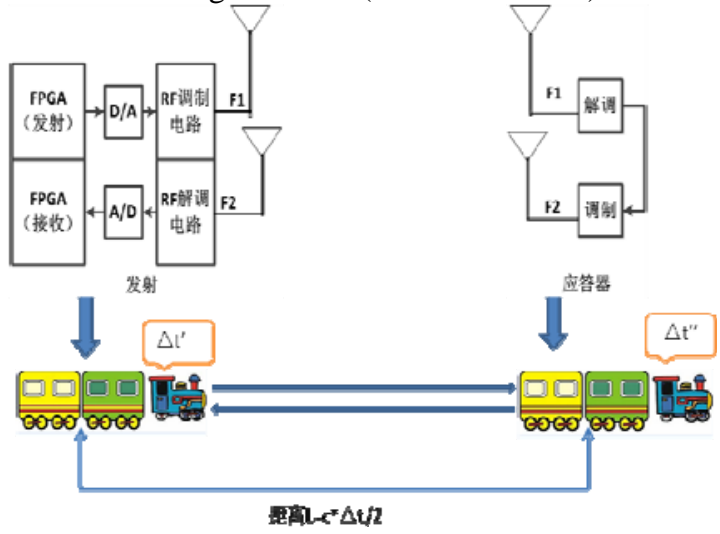

Figure 1. Principle of NLOS ranging radar

\section{B. Ranging Principle}

As is shown in Fig. 2, the phase of the sine signal varies linearly with time [4-7]. Therefore, we can get time t from the given phase $\varphi$ through $\frac{t}{T}=\frac{\varphi}{2 * \pi}$. As can be seen, the vertical line corresponds to four different phase values at the same time. Except the first harmonic wave, the phase values 
of other sub-carriers should add an integer multiple of $2 \pi$. That is how phase ambiguity exists.

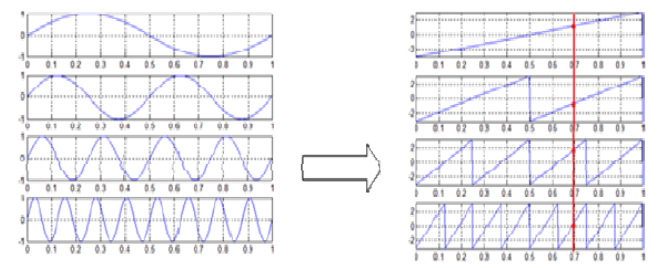

Figure 2. The relationship between OFDM sub-carrier and phase

\section{Parameter Design at the Transmitter End}

- The design distance should be $1.5 \mathrm{~km}$ because of the real distance between two stations.

- Suppose the phase delay is restricted to $2 \pi$, then the fundamental frequency should be lower than 100 $\mathrm{KHz}$.

- We choose f, 2f, 4f, 8f harmonic waves in terms of algorithm requirement.

\section{NLOS RADAR SYSTEM}

Fig 3 shows the phase of each sub-carrier changes over time in one period. We assume that, the phase of the highest harmonic is in the range of $0 \sim 2 \pi$.

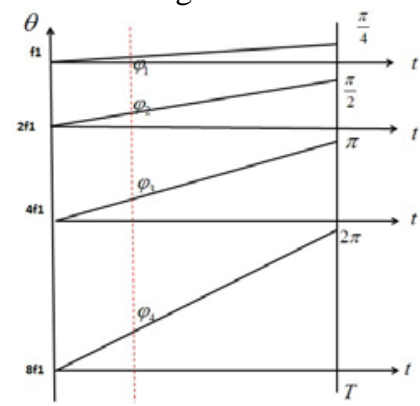

Figure 3. Frequency of sub-carrier

The dotted line illustrates an arbitrary measurement result. Then, the distribution of each harmonic wave is shown as follows:

$$
\hat{\varphi}_{1} \sim\left(\frac{\theta_{0}}{8}, \sigma_{0}^{2}\right) \hat{\varphi}_{2} \sim\left(\frac{\theta_{0}}{4}, \sigma_{0}^{2}\right) \hat{\varphi}_{3} \sim\left(\frac{\theta_{0}}{2}, \sigma_{0}{ }^{2}\right) \hat{\varphi}_{4} \sim\left(\theta_{0}, \sigma_{0}^{2}\right)
$$

We can see that they satisfy the geometric mean value and equal variance distrubution . More generally, we assume $\widehat{x}_{i} \sim N\left(a_{i} \theta_{0}, \sigma_{0}{ }^{2}\right), a_{i}$ is arbitrary. We should find the suitable value $y$ according to the vlue $x_{i}$ which also satisfies $E(y)=\theta_{0}$ and minimize the value $D(y)$. Now we list the following methods:

\section{A. Equal Variance Method}

Equal variance method is actually the equally weighted processing. We should guarantee that $E(y)=\theta_{0}$ by directly adding up or subtracting off $x_{i}$ and then dividing a constant. Two methods are as follows:

a) Equal Variance subtraction(Normal

OFDM measurement uses this method)

$$
\begin{gathered}
\text { Let } y=\frac{1}{a_{1}-a_{2}}\left(x_{1}-x_{2}\right) \text {, we have } \\
D(y)=\frac{2 \sigma_{0}^{2}}{\left(a_{1}-a_{2}\right)^{2}}
\end{gathered}
$$

b) Equal Variance addition

$$
\begin{aligned}
& \text { Let } y=\frac{1}{\sum_{i=1}^{n} a_{i}} \sum_{i=1}^{n} x_{i} \text {, we have } \\
& D_{1}(y)=\frac{1}{\left(\sum_{i=1}^{n} a_{i}\right)^{2}} n \sigma_{0}^{2}=\frac{n \sigma_{0}^{2}}{\left(\sum_{i=1}^{n} a_{i}\right)^{2}}
\end{aligned}
$$

Since the value of $a_{i}$ is positive, we compare (1) (2) and find that equal variance addition acquires lower variance which means more accurate values.

\section{B. Equal Mean Value Method}

Transferring the geometric mean value distribution into equal mean value distribution by dividing corresponding coefficient is so called equal mean value method.

a) Equal Mean Value Equal Coefficient

$$
\begin{gathered}
\text { Let } y=\sum_{i=1}^{n} k_{i} y_{i}, \quad \sum_{i=1}^{n} k_{i}=1 \text {. So } \\
E_{2}(y)=E_{2}\left(\sum_{i=1}^{n} k_{i} y_{i}\right)=\theta_{0}, \quad D_{2}(y)=\sum_{i=1}^{n} k_{i}^{2} \frac{\sigma_{0}^{2}}{a_{i}^{2}}
\end{gathered}
$$

The principle of equal mean value coefficient is equalizing all $k_{i}$ on condition of $\sum_{i=1}^{n} k_{i}=1$, i.e. $k_{i}=\frac{1}{n}$.

$$
D_{2}(y)=\sum_{i=1}^{n} k_{i}^{2} \frac{\sigma_{0}^{2}}{a_{i}^{2}}=\frac{\sigma_{0}^{2}}{n^{2}} \sum_{i=1}^{n} \frac{1}{a_{i}^{2}}
$$

b) Equal Mean Value Weighted Coefficient

The principle of equal mean equal weighted coefficient is finding proper $k_{i}$ in order to minimize $D_{3}=\sum_{i=1}^{n} k_{i}{ }^{2} \sigma_{i}^{2}$. Suppose $z=\sum_{i=1}^{n} k_{i}^{2} \sigma_{i}^{2}+\lambda\left(\sum_{i=1}^{n} k_{i}-1\right)$ has extreme value, i.e. $\frac{\partial z}{\partial k_{i}}=2 k_{i} \sigma_{i}^{2}+\lambda=0$. So $k_{i} \sigma_{i}^{2}$ is a constant. 


$$
\begin{array}{r}
k_{1}: k_{2}: k_{3}: \ldots: k_{n}=\frac{1}{\sigma_{1}^{2}}: \frac{1}{\sigma_{2}^{2}}: \frac{1}{\sigma_{3}^{2}}: \ldots: \frac{1}{\sigma_{n}^{2}} \\
k_{j}=\frac{1}{D\left(y_{j}\right) \sum_{i=1}^{n} \frac{1}{D\left(y_{i}\right)}}=\frac{a_{j}^{2}}{\sum_{i=1}^{n} a_{i}^{2}} \\
D_{3}(y)=\sigma_{0}^{2} \sum_{i=1}^{n} \frac{a_{i}^{2}}{\left(\sum_{j=1}^{n} a_{j}^{2}\right)^{2}}=\sigma_{0}^{2} \frac{1}{\sum_{i=1}^{n} a_{i}^{2}}
\end{array}
$$

c) Comparison of the Methods

From the above derivation we know that the variance of equal mean value weighted coefficient method is the extreme value of equal value method, so $D_{3}(y)<D_{2}(y)$. Now we just need to compare $D_{1}(y)$ and $D_{3}(y)$.

$$
\begin{aligned}
& \text { Suppose } p=\frac{n}{\left(\sum_{i=1}^{n} a_{i}\right)^{2}}, \quad q=\frac{1}{\sum_{i=1}^{n} a_{i}^{2}} \\
& n\left(\frac{1}{q}-\frac{1}{p}\right)=\sum_{j=2}^{n} \sum_{i=1}^{j-1}\left(a_{i}-a_{j}\right)^{2} \geq 0 \\
& \text { So, } D_{1}(y) \geq D_{3}(y)
\end{aligned}
$$

So equal mean value weighted coefficient method can get the most accurate result.

\section{THE FOUR-WIRE CARRIER MEASUREMENT ERRORS}

Suppose the four-wire carrier coefficients are as follows:

$$
a_{1}=\frac{1}{8}, a_{2}=\frac{1}{4}, a_{3}=\frac{1}{2}, a_{4}=1 .
$$

Insert them into the following three variance formula.

A. Equal Variance Addition Method

Insert into (2) , we have

$$
D_{1}(y)=\frac{4 \sigma_{0}^{2}}{\left(\frac{1}{8}+\frac{1}{4}+\frac{1}{2}+1\right)^{2}}=\frac{256}{225} \sigma_{0}^{2}
$$

\section{B. Equal Mean Value Equal Coefficient Method}

Insert into (4), we have

$$
D_{2}(y)=\frac{1}{4^{2}}\left(8^{2}+4^{2}+2^{2}+1\right) \sigma_{0}^{2}=\frac{85}{16} \sigma_{0}^{2}
$$

\section{Equal Mean Value weighted Coefficient Method} Insert into (8) we have

$$
D_{3}(y)=\frac{\sigma_{0}^{2}}{\left(1+\frac{1}{2^{2}}+\frac{1}{4^{2}}+\frac{1}{8^{2}}\right)}=\frac{64}{85} \sigma_{0}^{2} .
$$

\section{Result Analysis}

From the above calculation we know that

$$
\sqrt{D_{3}}=0.376 \sqrt{D_{2}}=0.813 \sqrt{D_{1}}
$$

Obviously the equal mean value weighted coefficient method is the optimal data processing method.

\section{THE RELATIONSHIP BETWEEN VARIANCE AND SNR}

As shown in Fig.4, when transmitting in the channel, OFDM signal $s_{i}$ added the noise $n_{i}\left(n_{i} \sim N\left(0, \sigma_{0}{ }^{2}\right)\right)$.

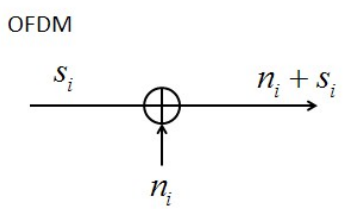

Figure 4. Sine signal superposition with narrow band Gauss- noise

We consider the signal as the superposition of sine and narrowband Gaussian noise, then the probability density function [8] is as follows:

$$
f\left(\varphi-\theta_{0}\right)=\frac{1}{2 \pi} e^{-\gamma}\left\{1+\sqrt{\pi \gamma} \cos \left(\varphi-\theta_{0}\right) \cdot e^{\gamma \cos ^{2}\left(\varphi-\theta_{0}\right)}\left[1+\operatorname{erf}\left(\sqrt{\gamma} \cos \left(\varphi-\theta_{0}\right)\right)\right]\right\}
$$

$\gamma$ is SNR, namely the ration of signal average power and Gauss narrow band signal average power. We can see from Fig. 5 that with the increase of $\gamma$, the phase random variable range decreases and finally approaches zero (that is the phase of the signal itself). However as $\gamma$ approaches zero, it tends to conform to the uniform distribution.

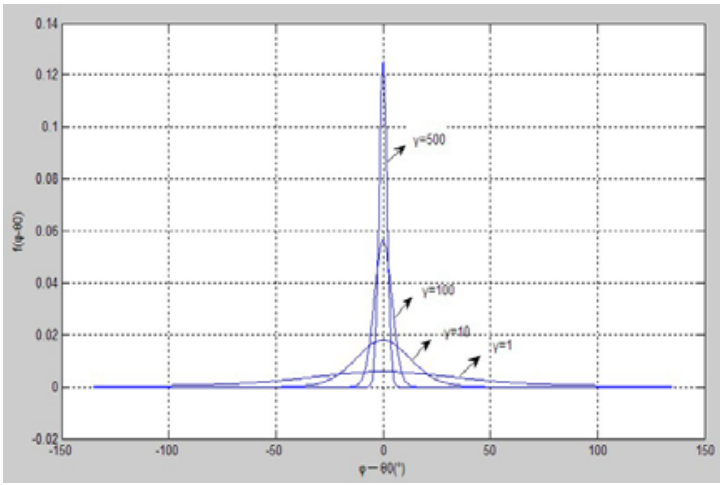

Figure 5. Phase distribution of Sine signal superposition with narrow band Gauss- noise

We can get the mean value and variance based on the above distribution graph, the formula is as follows:

$$
\begin{gathered}
E\left(\varphi-\theta_{0}\right)=\operatorname{sum}\left(\left(\varphi-\theta_{0}\right) f\left(\varphi-\theta_{0}\right)\right) \\
D\left(\varphi-\theta_{0}\right)=\operatorname{sum}\left(f\left(\varphi-\theta_{0}\right)\left(\varphi-\theta_{0}-E\left(\varphi-\theta_{0}\right)\right)^{2}\right)
\end{gathered}
$$

TABLE I. VARIANCE VERSUS SNR

\begin{tabular}{|c|c|c|c|c|}
\hline SNR $\boldsymbol{\gamma}$ & $\mathbf{1}$ & $\mathbf{1 0}$ & $\mathbf{1 0 0}$ & $\mathbf{5 0 0}$ \\
\hline Variance $\sigma_{0}^{2}$ & $43.325^{2}$ & $13.185^{2}$ & $4.0617^{2}$ & $1.8128^{2}$ \\
\hline
\end{tabular}


Fig.6 illustrates the relationship between standard variance and SNR.

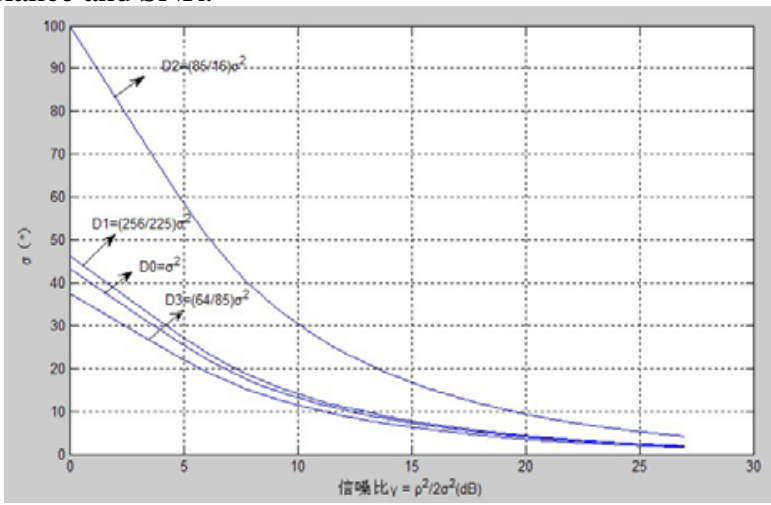

Figure 6. Angle standard variance versus SNR

Change the vertical coordinate into distance standard variance and we can get the relationship between distance standard variance and SNR, which is shown in Fig.7.

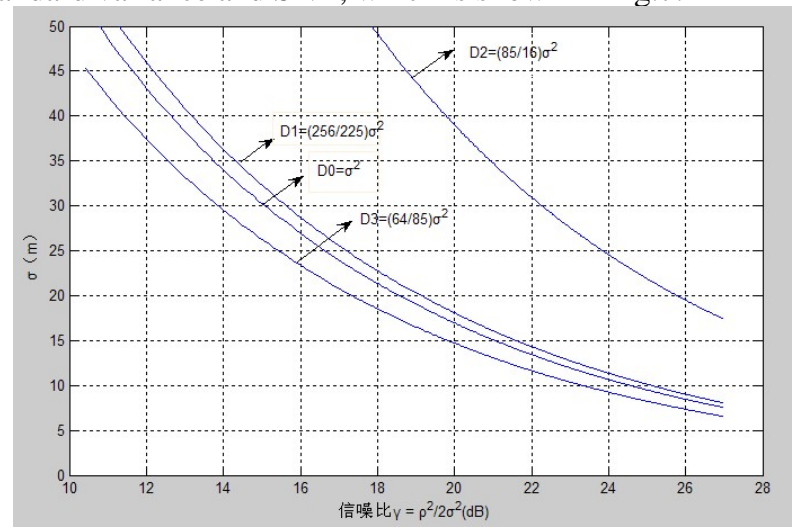

Figure 7. Distance standard variance versus SNR

We can see from Fig. 7 that equal mean value weighted coefficient method acquires the minimum measuring accuracy in condition of the same SNR. When $\gamma=10 \mathrm{~dB}$, the minimum measuring distance standard variance is $45 \mathrm{~m}$; when $\gamma=20 d B$, the minimum measuring distance standard variance is $15 \mathrm{~m}$; when $\gamma=27 d B$, the minimum measuring distance standard variance is $7 \mathrm{~m}$. As for the NLOS ranging in the tunnel, this result is satisfactory.

\section{CONCLUSION}

This article proposes a NLOS radar ranging system based on OFDM waveform harmonic phase measurement principle. According to the specific requirements of subway ranging, we choose the fundamental frequency of $100 \mathrm{kHz}$ and the 2th, 4th, 8th harmonic waves. Then we compare different data processing methods. The first is equal variance method which can be divided into subtraction (now been used) and addition (a lower variance). We also have the equal mean value method, which can be divided into two methods: direct and weighted adding. By comparing the different variances, we find that measurement error of equal mean value weighted is only 0.376 of equal mean value adding and 0.813 of equal variance and is the optimal method. Moreover, when the successive SNR value is $10 \mathrm{~dB}, 20 \mathrm{~dB}$ and $27 \mathrm{~dB}$, the minimum measuring distance respectively equals to $45 \mathrm{~m}, 15$ and $7 \mathrm{~m}$. As for the NLOS ranging in the tunnel, this result is satisfactory.

\section{ACKNOWLEDGMENT}

This work is supported by Shanghai Leading Academic Discipline Project S30108, NSFC 61132003 and NSFC 61171086.

\section{REFERENCES}

[1] Jianhui Wang. A New Approach for Cellular Wireless Location In NLOS Situations, Communications and Networking ,China , Oct., 2527, 2009, Conference Publications.

[2] Mazuelas, S. Prior NLOS Measurement Correction for Positioning in Cellular Wireless Networks, Vehicular Technology, 2009, 58, 58(5):2585-2591.

[3] Guangliang Ren, Chuiqiang Sun, Space Precision OFDM Ranging System. Journal of Xidian University, v 36, n 2, 2009.

[4] Xingbin Cao, Research of the OFDM Modulation and Demodulation Technology Based on 802.16e. Communication Technology, 2011,(2).

[5] Sen, Satyabrata, Target detection in clutter using adaptive OFDM radar. IEEE Signal Processing Letters, v 16, n 7, p 592-595, 2009.

[6] Mohseni, Reza, Multicarrier constant envelope OFDM signal design for radar applications.AEU - International Journal of Electronics and Communications, v 64, n 11, p 999-1008, November 2010.

[7] Sen, Satyabrata, Tang, Gongguo, Nehorai, Arye. Multiobjective optimization of OFDM radar waveform for target detection. IEEE Transactions on Signal Processing, v 59, n 2, p 639-652, February 2011.

[8] Xiaoqin Yu, Hengkai Zhao, Communication Theory-_-The Relevant Technology of the Information Transmission. Beijing: TsingHua University Press, 2007. 